\title{
Epicardial screw-in sensing lead on the left ventricle to treat undersensing of ventricular arrhythmias in a patient with arrhythmogenic right ventricular cardiomyopathy
}

Maciej Kempa ${ }^{1}$, Grzegorz Łaskawski ${ }^{2}$, Szymon Budrejko ${ }^{1}$, Tomasz Królak ${ }^{1}$, Dariusz Kozłowski ${ }^{1}$, Jan Rogowski ${ }^{2}$, Grzegorz Raczak ${ }^{1}$

${ }^{1}$ Clinic of Cardiology and Electrotherapy, $2^{\text {nd }}$ Chair of Cardiology, Medical University of Gdansk, Poland

${ }^{2}$ Chair and Clinic of Cardiac and Vascular Surgery, Medical University of Gdansk, Poland

A 37-year-old patient, with a history of arrhythmogenic right ventricular cardiomyopathy (ARVC) and involvement of the left ventricle, was admitted due to repetitive implantable cardioverter-defibrillator (ICD) shocks and syncope in the course of ventricular tachycardia (VT) and ventricular fibrillation (VF).

He had previously experienced multiple appropriate therapies for VT, and had undergone endocardial/epicardial ablation procedures with

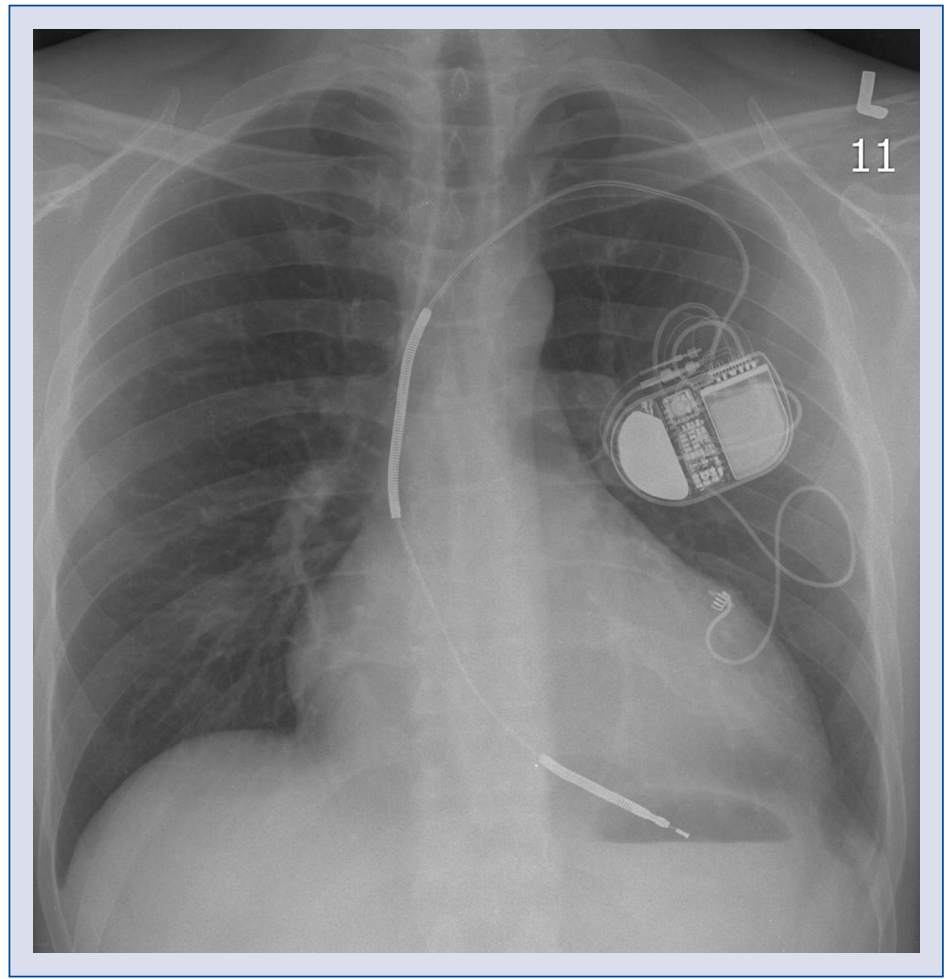

Figure 1. Chest X-ray showing final implantable cardioverter-defibrillator system after the procedure, standing position, postero-anterior projection.

Address for correspondence: Dr. Szymon Budrejko, $2^{\text {nd }}$ Chair of Cardiology, Department of Cardiology and Electrotherapy, Medical University of Gdansk, ul. Dębinki 7, 80-211 Gdańsk, Poland, tel: +48583493910, fax: +48583493920, e-mail: budrejko@gumed.edu.pl

Received: 26.04.2017 Accepted: 09.07.2017 
a temporary reduction of arrhythmia burden. He had been previously implanted with an ICD (dualcoil passive-fixation lead). Prior treatment with sotalol and amiodarone was not successful.

Implantable cardioverter-defibrillator interrogation showed intermittent under-sensing of VT and VF, which lead to a delay in therapy. R-wave amplitude during sinus rhythm was $2-3 \mathrm{mV}$.

The initial plan of treatment was to exchange the right ventricular (RV) ICD lead, in order to achieve appropriate R-wave amplitude and sensing. After reviewing voltage maps from previous ablation procedures - RV voltage maps showed RV potentials of over $4 \mathrm{mV}$ only in the area around the His bundle, with the rest of the RV being scarred.
Epicardial maps showed higher potential amplitude only over the lateral and posterior basal surface of the left ventricle. Therefore it was decided to implant an epicardial sensing lead in the region with sufficient signal amplitude. An epicardial lead was implanted from left micro-thoracotomy approach (R-wave amplitude $20 \mathrm{mV}$, threshold $0.8 \mathrm{~V} / 0.4 \mathrm{~ms}$, impedance $800 \mathrm{Ohm}$ ) and connected instead of the endocardial pace-sense channel, together with highvoltage channels of the previously implanted lead, to the ICD (Fig. 1). During follow-up, the patient experienced VT and VF episodes that were appropriately detected and treated with antytachycardia pacing or shocks. This approach has proven feasible, when the conventional ICD system cannot be applied.

Conflict of interest: None declared 